\title{
Changing patterns of burn infections.
}

\author{
B Fouzia ${ }^{1}$, A S Damle ${ }^{2}$, G Maher ${ }^{3}$. \\ ${ }^{I}$ (Department of Microbiology, Shadan Institute Of Medical Sciences Hyderabad, NTRUHS, India) \\ ${ }_{2}^{2}$ (Department of Microbiology, Govt. Medical College Aurangabad, MUHS, India) \\ ${ }^{3}$ (Department of Microbiology, Govt. Medical College Aurangabad, MUHS, India)
}

\begin{abstract}
Purpose: The present study was aimed to compare the bacterial isolates and their antibiotic susceptibility pattern over a period of time. Materials and Methods: Pus swabs were collected from burns, Gram stained and cultured aerobically. Bacterial isolates were identified and antibiotic susceptibility tests were performed. Results: Staphylococcus aureus was the predominant organism isolated with Gentamicin and Methicillin resistance. Pseudomonas aeruginosa showed Meropenem resistance, Enterobacteriaceae family showed 32\% ESBL(Extended Spectrum Beta Lactamase]) and 16\% MBL(Metallo Beta Lactamases). Conclusion:High resistance to Methicillin and high prevalence of ESBL \& MBL producing strains.
\end{abstract}

Keywords: Antibiotic resistance, Burn, ESBL, MBL.

\section{Introduction:}

In India 700,000 burn patients are admitted to hospitals each year. Infection is a major cause of morbidity and mortality among burn patients. $75 \%$ of all deaths following burns are related to infection. There is a higher rate of nosocomial infections in burn wards [1]

In patients of burns, the resistance is already lowered due to multiple factors and slough acts as a good hospital medium for the growth of bacteria, especially in case of deep burns [2]

The introduction of widespread use of chemotherapeutic and antibiotic agents has resulted in the profound changes in the number and character of infections that are being encountered. [3]

There is a sequence of different bacterial types and of numbers of bacteria present on the surface of the wound depending on the age and physiological status of the burn eschar. Shortly after the wound occurs, Gram positive bacteria in low numbers tend to colonize on the surface. As the eschar ages colonization occurs with Gram negative organisms in high concentrations, but as the eschar sloughs off Gram positive organisms reappear [4]

The present study was aimed to compare the bacterial isolates and their antibiotic susceptibility pattern over a period of time.

\section{Materials and Methods:}

The study was carried out in a tertiary care hospital, Aurangabad, from March 1993 - April 1994 and January 2012 to June 2012. Study population included all patients admitted to the burn unit in our hospital.

The pus was collected on two plain sterile swabs and transported to the laboratory immediately [5]. One of the swabs was used for making smear and the other for inoculating culture media. The smears were stained with Gram stain and findings noted down. Each specimen was inoculated on a plate of sheep blood agar and Mac Conkeys media. Both the plates were incubated at $37^{\circ} \mathrm{C}$ aerobically overnight.

Next day the plates were examined and colony characters noted down. Bacterial pathogens were identified by conventional methods. Antimicrobial susceptibility tests were performed as per CLSI guidelines [6]. "Tables"( $1,3,4,6)$

Whenever warranted second, third and fourth or more number of samples were collected at 7 .

10 days interval and the samples were processed in the same way as mentioned above. "Table"(2)

In the first study 150 cases of burns were included. 67 males and 83 females in the age group of $1-70$ years. The youngest patient was 14 months old and the oldest patient was 70 years old. The percentage of burns ranged from 6\% - 86\%. 89 patients had superficial to deep burns, 37 had superficial and 24 patients had deep burns.

In the second study total number of samples tested were 136. Culture positive 102(75\%) and culture negative $34(25 \%)$. 


\begin{tabular}{|l|lllllll|}
\hline Table 1: Different & organisms isolated in I study \\
\hline Total Number & \multicolumn{2}{l|}{ Gram } & Positive & \multicolumn{4}{l|}{ Gram Negative } \\
\hline S.aureus & CoNS & Strep & Kleb & Pseudo & E.coli & Proteus & \\
\hline 266 & 102 & 18 & 1 & 56 & 43 & 28 & 18 \\
\hline$\%$ & 38.36 & 6.76 & 0.37 & 21.05 & 16.16 & 10.54 & 6.76 \\
\hline
\end{tabular}

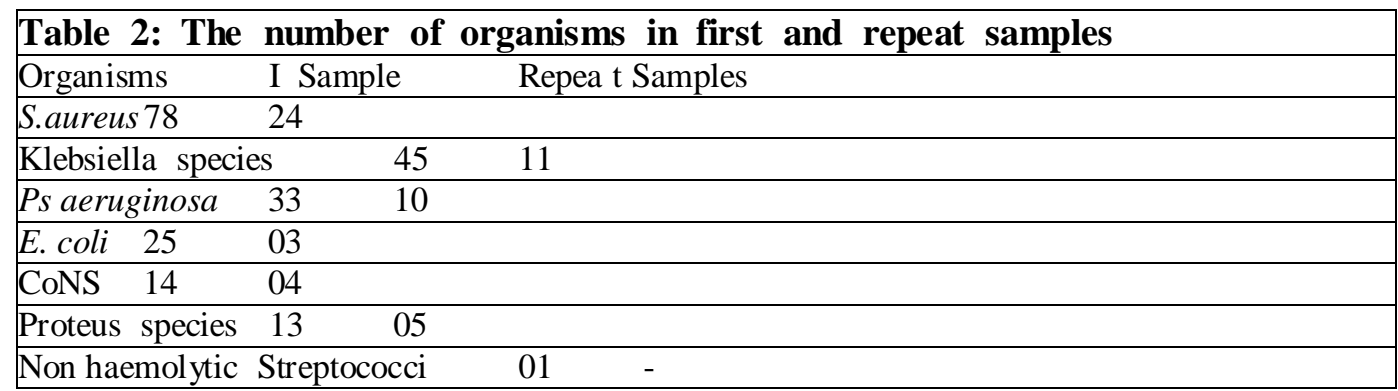

Table 3: Percentage of antibiotic resistance pattern of isolates in the first study ISOLATED ANTIBIOTIC DISC



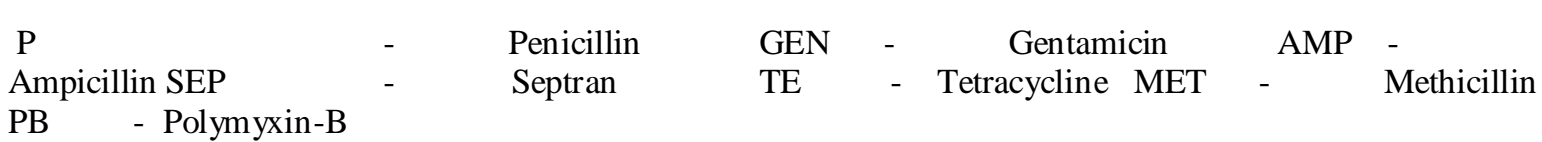

Table 4: Bacterial pathogens isolated from study 2

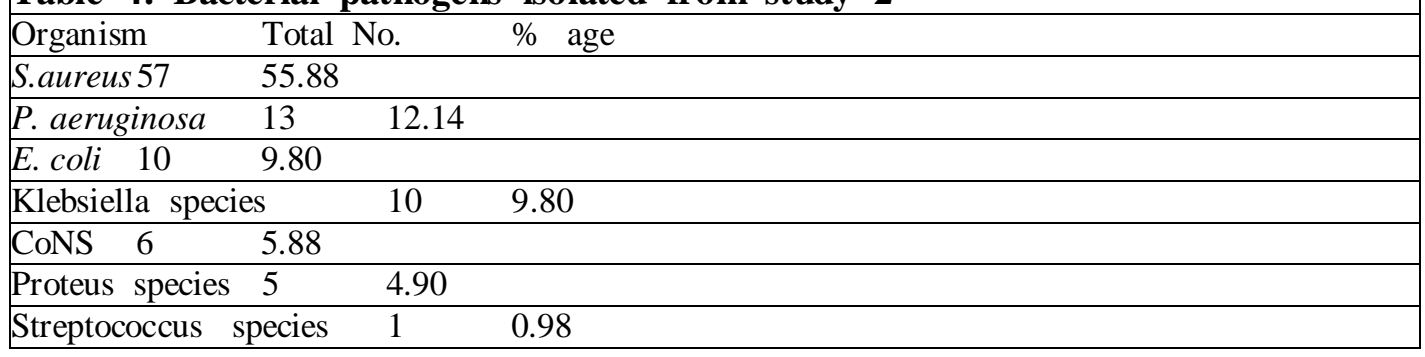

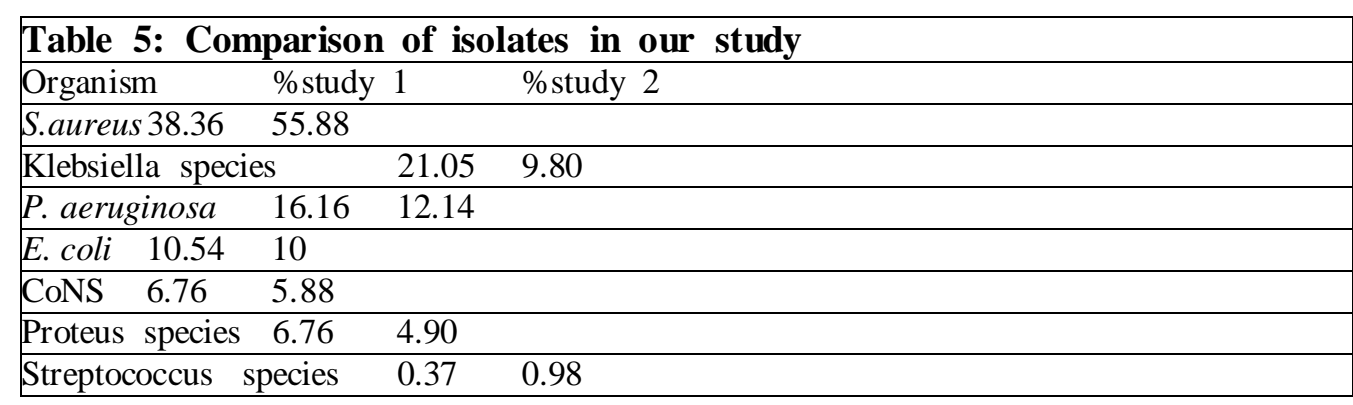




\begin{tabular}{|c|c|c|c|c|c|c|}
\hline \multicolumn{7}{|c|}{ Table 6: Percentage of antibiotic resistance pattern of isolates in study 2} \\
\hline Isolate GEN & AMP & COT & $\mathrm{CD}$ & TE & AMC & CIP \\
\hline S.aureus 73.68 & 95.23 & 79.59 & 62.5 & 10.52 & 60.46 & 77.41 \\
\hline P. aeruginosa & - & - & - & - & - & 88.88 \\
\hline Enterobacteriaceae 57.14 & - & 81.82 & - & - & - & 69.23 \\
\hline Isolate $\mathrm{AK} \quad \mathrm{CPM} \quad \mathrm{CAZ}$ & & $\mathrm{CAC}$ & CTX & CX PI & PIT & MRP \\
\hline S.aureus- & - & - & - & 21.42 & - & - \\
\hline P.aeruginosa & 88.88 & 76.93 & - & 72.72 & - & - 33.3415 .38 \\
\hline Enterobacteriaceae & - & 63.15 & 70 & 24.86 & 87.50 & $22.22-16.66$ \\
\hline
\end{tabular}

GEN-Gentamicin,

E - Erythromycin,

COT - Co- Trimoxazole, CD - Clindamycin, TE-Tetracycline, AMC - Amoxycillin-Clavulanic acid, CIP - Ciprofloxacin, AK - Amikacin, CPM - Cefipime, CAZ - Ceftazidime, Ceffazidime Clavulanic acid, CTX - Cefotaxime CX - Cefoxitin, Piperacillin, PIT - Piperacillin/Tazobactum, PI Meropenem

\section{Discussion:}

Over the past two decades the prominent organisms causing infection in burn patients have changed dramatically. Gram negative opportunistic organisms have receded in importance in recent years, Staphylococcus aureus being the most frequent infecting organism [7]. Which is also the case in our study. "Table" (5)

Staphylococcus aureus: Staphylococcus aureus in burn patients is usually resistant to various antibiotics, from which it can be inferred that they are acquired in the hospital [8]. Staphylococcal isolates have varied from $20 \%-80 \%$ as demonstrated by various workers $[1,9,10,11,12,13,20]$. Blactamase production was shown to be $97.9 \%$ in our institute in 1992 [14], which was increased to $100 \%$ in our study in 1993 - 1994.

Coagulase Negative Staphylococci(CoNS): CoNS have gained increasing importance in burns as the pathogen mostly causing the destruction of already healed grafts in a later phase of the treatment [15]. Slime is important in the colonization of CoNS and may act as a virulence factor in infections produced by these organisms [16]. In both of our studies CoNS accounted for 6\%. CoNS were tested for slime production and all were found to be slime producers which clearly indicate their role as pathogens.

Pseudomonas aeruginosa: Lowbury demonstrated that Pseudomonas infections are probably a result of hospital contamination, when they found the same serologic type prevalent in burns of patients and in their hospital environment [8]. Pseudomonas infections in burn patients range from 6 $80 \%[1,9,10.11,12,17,18,20]$. In our study it has reduced from $16 \%$ to $12 \%$ which shows measures are being taken to reduce HAI [Hospital Acquired Infection].

Klebsiella species: The isolation of Klebsiella species from burn wounds has varied from $0.5 \%$ - $29 \%[9,11,13,19,20]$. In our study there is again decrease from $21.05 \%-9.80 \%$.

Escherichia coli: Isolation of E.coli varied from $6 \%-35 \%[9,11,18]$. In our study it has remained constant at $10 \%$ even after two decades !

Proteus species: Proteus isolates varied from $2.3 \%$ - 9\% $[9,13,20]$. In our study it has reduced from $6.76 \%-4.90 \%$.

Streptococcus species: Streptococcus isolates have varied from $0 \%-20.5 \%[9,10,19]$. In our both studies one isolate each was found.

The low incidence of streptococci in burn wound has been explained by Lowbury by the fact that Streptococci though at times common in burn unit may disappear absolutely for few years only to re-appear [8].

This statement of Lowbury is true in its correct sense, such that a study carried out in our institute comprised $9.5 \%$ of streptococci [9]. whereas in the present study it has decreased to a single isolate. Will it re-appear again is yet to be seen.

\section{Results:}

Multiple antibiotic resistance in the infecting agents has for many years presented difficulties in the treatment of burn patients. The strains in different studies are showing different patterns of antibiotic resistance. It may be because of difference in antibiotics used for treatment at various 
hospitals.

In the first study Staphylococcus aureus showed 61\% resistance to Methicillin and same 61\% were also resistant to Gentamicin making them GMRSA (Gentamicin Methicillin

Resistant Staphylococcus aureus). In the second study Staphylococcus aureus showed cefoxitin resistance $21.42 \%$ indicating high prevalence of MRSA(Methicillin resistant Staphylococcus aureus) and Gentamicin resistance $73.68 \%$.

Tetracycline resistance became common in our institute as is evident by the study of sengupta et al. They have reported $92.03 \%$ resistance[9]. Probably because of this, the use of tetracycline declined in clinical practice. This in turn might have led to decrease in resistance to tetracycline which is evident from our studies which has further decreased from 79\% - 52\%. In contrast to tetracycline, all other antibiotics tested by us showed sizeable number of resistant strains.

Pseudomonas aeruginosa showed meropenem resistance (15.38\%) indicating MBL (Metallo beta lactamase) incidence phenotypically.

Enterobacterisceae family showed 32\% ESBL(Extended spectrum beta lactamase) producer, $16 \%$ MBL producer.

ESBL was not very common among those days, hence not studied in the first study.

To conclude, Staphylococcus aureus is the predominant isolate even in repeat samples with high prevalence of MRSA in burn unit. High prevalence of ESBL and MBL producing strains. Antibiotic policy should be strictly adhered to and followed by the clinicians and indiscriminate use of antibiotics should not be practised.

\section{References}

[1]. AC Ganguli . Burns. J ind Med Asso. 1976; 67: 150 -- 152.

[2]. L Colebrook. The control of infection in burns. The Lancet i:1948; $893-899$.

[3]. M Finland, WF Jones, MW Barnes . Occurence of serious bacterial infection since introduction of antibacterial agents.JAMA 1959; 24: 2188.

[4]. HP Dalton and HC Nottebart. Burn wounds, In interpretative Medical microbiology. London. ChurchillLivingstone.1986; 671 -- 672.

[5]. M Cheesbrough . Collection, transport and examination of pus from wound, abscesses, burns and sinuses, In Medical laboratory manual for tropical countries. Oxford : ELBS. Tropical health technology/ Butterworth-Heinemann $1991 ;$ vol.2 : 124 -- 129

[6]. Clinical and laboratory standards institute performance standards for antimicrobial susceptibility testing. Twenty first informational supplement M100 -- S21. Wayne, PA; 2011. P 48 - 49, 68 -- 76.

[7]. BA Pruitt, MC Colonel, AT Manus . Opportunistic infections in severely burned patients. Am J Med 1984; 76(3A): 146 -- 153 .

[8]. EJL Lowbury . Infection associated with burns. Post Graduate Med J 1972; 48 : 338 -- 341

[9]. SR Sengupta , MP Bansal, PK Deshpande , KD Sharma. Infection of burns. Ind J Surg 1989; 34: 327 -- 333.

[10]. VK Sharma, DS Agarwal, Satyanand. Bactriological study of infection in patients with burns. Ind J Med Res 1981;73: $697-709$.

[11]. OJ Szab, Belicza, R Edal , F Rozgonyi . Distribution and antibiotic resistance of aerobic bacteria isolated from infected wounds caused by burns. Orv Hetil Mar 1991; 132 (13): 687 -- 690.

[12]. CB Tomanovi, CZ Hadzi, CB Jokovi , CD Bozinovi, Nanusevi CO, CV Mirovi . Vojnosanit Pregl 1993; (1): 39 42. [13]. YP Zang. Common pathogens in burn infection and changes in their drug sensitivity. Chung Hua Cheng HsingShaoShang Wai KO Tsa Chih 1991; 7(2) : 108 -- 110 ( English abstract).

[14]. SD Bhat, AB Deshmukh , AS Damle , RP karyakarte. Incidence of beta lactamase producer strains ofStaphylococcus aureus and their biological properties. Indian Medical Gazette 1991; 346 -- 348.

[15]. D Kistler, N Jahns, K Pichulla , R Hettich. Pathogen spectrum and pathways of infection in an intensive care burnunit. Anasth intensivther Notfallmed June 1990; 25 (3) : 216 -- 221 (English abstract).

[16]. N Nayak , N Pal, A Ayyagari . Slime production as a marker of coagulase negative staphylococcal infection with/without artificial devices. Ind L Med Micro 1990; 8(3) : 92 -- 95.

[17]. M Gore , HK Shah , N Saileshwar . Burning injury in children. Ind pract Dec 1988; 847 -- 850.

[18]. LS Nakhla and R Beta Sanders. Microbiological aspects of burns at Mount Varnon hospital UK. Burns 1991; 17(4) :309 -- 312 .

[19]. R Bharadwaj . Bacteriology of burn wound using the qualitative full thickness biopsy technique. Ind Med Res 1933; $78 ; 337$.

[20]. M Mehta , P Datta, V Gupta . Bacterial isolates from burn wound infections and their antibiograms : A eight year study.Indian J plast surg 2007; $40: 25$-- 8 . 